\title{
The expression of epithelial-mesenchymal transition-related proteins in biliary epithelial cells is associated with liver fibrosis in biliary atresia
}

Yongtao Xiao ${ }^{1-3}$, Ying Zhou ${ }^{1,3}$, Yingwei Chen ${ }^{2,3}$, Kejun Zhou ${ }^{2,3}$, Jie Wen ${ }^{2,3}$, Yang Wang ${ }^{2,3}$, Jun Wang ${ }^{1,3}$ and Wei Cai ${ }^{1-3}$

BACKGROUND: The epithelial-mesenchymal transition (EMT) has been implicated as a key mechanism in the pathogenesis of liver fibrosis. The miR-200 family has been shown to inhibit EMT.

METHODS: Liver fibrosis levels were assessed with Masson's trichrome staining of liver samples obtained from biliary atresia (BA) patients. The expressions of cytokeratin-7 (CK-7) and $\alpha$-smooth muscle actin ( $\alpha$-SMA) in the liver sections were detected by immunohistochemical and immunofluorescent staining. EMTs were induced by transforming growth factor (TGF)- $\beta 1$ in human biliary epithelial cells (BECs) in vitro.

RESULTS: We showed that the EMT-related proteins CK-7 and $\alpha$-SMA colocalized to the intrahepatic BECs in the liver sections of patients with BA. The level of $\alpha$-SMA expression was related to liver fibrosis stage in BA. EMT in primary human intrahepatic BECs was induced by TGF- $\beta 1$ in vitro. miR-200b is one member of the miR-200 family and significantly inhibited TGF$\beta 1$-mediated EMT in BECs.

CONCLUSION: Together, these data suggest that the occurrence of EMT in BECs might contribute to BA fibrosis. miR-200b significantly affects the development and progression of TGF$\beta 1$-dependent EMT and fibrosis in vitro.

B iliary atresia (BA) is an infantile neonatal disease that is characterized by ascending obstruction of the biliary tree associated with a progressive sclerosing cholangiopathy (1). The progression of hepatic fibrosis in BA is quite dramatic. Many BA patients already have cirrhosis within a few weeks after birth. However, the etiologies and molecular mechanisms that are involved in these expeditious fibrogenic processes have not been well established.

The epithelial-mesenchymal transition (EMT) refers to a process in which epithelial cells lose cell-cell contacts and the unique protein expression patterns of epithelia and acquire the phenotypic characteristics of mesenchymal cells or fibroblast markers (2,3). Iwano et al. (4) first demonstrated that EMT in tubular epithelia is an important cause of murine kidney fibrosis. This finding was followed by similar studies of the liver, which suggested that hepatocytes can undergo EMT in vivo (5). Cholangiocytes then quickly became the focus of EMT research with the finding of evidence of EMT in rodent livers (6). However, additional and rigorous data are still needed to define the role of cholangiocyte EMT in BA fibrosis.

The miR-200 family consists of five members that are organized into two clusters: miRs-200b/a/429 and miRs-200c/141. Several studies have shown that the miR-200 family can suppress tumor metastasis and invasion by inhibiting EMT (7-10). Transforming growth factor (TGF)- $\beta 1$ has been shown to drive EMT in many systems and is also likely to drive EMT in the liver (11). We previously demonstrated that miR-200b inhibits TGF- $\beta 1$-induced EMT in intestinal epithelial cells (12). Here, we found that miR-200b significantly suppressed TGF- $\beta 1$-induced-EMT in biliary epithelial cells (BECs), which suggests that miR-200b might be a potential candidate for ameliorating BA fibrosis.

\section{RESULTS}

The Expression of EMT-Related Proteins in BECs Is Associated With BA Fibrosis

A total of 18 children with BA were enrolled in this study. All patients exhibited elevated direct bilirubin, total bile acid, serum $\gamma$-glutamyltransferase, and alanine aminotransferase levels (Table 1). Periportal and bridging fibroses were observed in the majority of the collected specimens (Figure 1a). Based on Masson's trichrome staining, the degrees of liver fibrosis in all biopsies were classified into classes I, II, III, or IV (Figure 1b). We also found that fibroblast-like cells were present around the hyperplastic bile ducts (Figure 1c,d).

The evidence for EMT in BA has been reported previously (13-15). In the present study, we double-stained all liver sections with $\alpha$-smooth muscle actin ( $\alpha$-SMA), which is a reliable marker of myofibroblastic cells, and the ductular epithelium marker CK-7. Consistent with previous observations, we demonstrated that multiple $\alpha$-SMA-positive cells had accumulated around the hyperplastic cholangiocytes (Figure 2a,b). Simultaneously, $\alpha$-SMA emerged in many CK-7-positive ductular cells (Figure 2c,d). Of particular interest, the levels

'Department of Pediatric Surgery, Xin Hua Hospital, School of Medicine, Shanghai Jiao Tong University, Shanghai, China; ${ }^{2}$ Shanghai Institute of Pediatric Research, Shanghai, China; ${ }^{3}$ Shanghai Key Laboratory of Pediatric Gastroenterology and Nutrition, Shanghai, China. Correspondence: Wei Cai (caiw1978@163.com) 
Table 1. The diagnostic information of the patients with biliary atresia

\begin{tabular}{|c|c|c|c|c|c|c|c|c|}
\hline$N$ & Age (days) & $\begin{array}{l}\text { Total bilirubin } \\
(\mu \mathrm{mol} / \mathrm{l})\end{array}$ & $\begin{array}{l}\text { Direct bilirubin } \\
\quad(\mu \mathrm{mol} / \mathrm{l})\end{array}$ & $\begin{array}{c}\text { Total bile } \\
\text { acids }(\mu \mathrm{mol} / \mathrm{l})\end{array}$ & $\begin{array}{c}\text { Alanine } \\
\text { aminotransferase (U/l) }\end{array}$ & $\begin{array}{c}\text { Aspertate } \\
\text { aminotransferase }(\mathrm{U} / \mathrm{I})\end{array}$ & $\begin{array}{c}\gamma \text {-Glutamyl } \\
\text { transferase (U/I) }\end{array}$ & $\begin{array}{l}\text { Fibrosis } \\
\text { grade }\end{array}$ \\
\hline 4 & $59 \pm 8.7$ & $137.8 \pm 8.1$ & $97.5 \pm 6.4$ & $126 \pm 52$ & $132.7 \pm 43.8$ & $225 \pm 109.7$ & $863 \pm 520.5$ & 1 \\
\hline 7 & $70.4 \pm 12.1$ & $185 \pm 59$ & $135.4 \pm 42.5$ & $180.5 \pm 80$ & $155 \pm 89.6$ & $251 \pm 105$ & $981 \pm 911$ & III \\
\hline 3 & $107 \pm 36.1$ & $133.7 \pm 22.5$ & $87.3 \pm 15$ & $90.5 \pm 9.2$ & $169 \pm 123$ & $361.6 \pm 238$ & $1,506 \pm 454.6$ & IV \\
\hline
\end{tabular}

All values are expressed as means \pm SD. Reference range of serum total bilirubin $(3.42-20.52 \mu \mathrm{mol} / \mathrm{l})$, direct bilirubin $(0-6.8 \mu \mathrm{mol} / \mathrm{l})$, total bile acids $(0-10 \mu \mathrm{mol} / \mathrm{I})$, alanine aminotransferase $(0-75 \mathrm{U} / \mathrm{l})$, aspertate aminotransferase (8-38 U/l), and $\gamma$-glutamyl transferase $(16-73 \mathrm{U} / \mathrm{l})$.
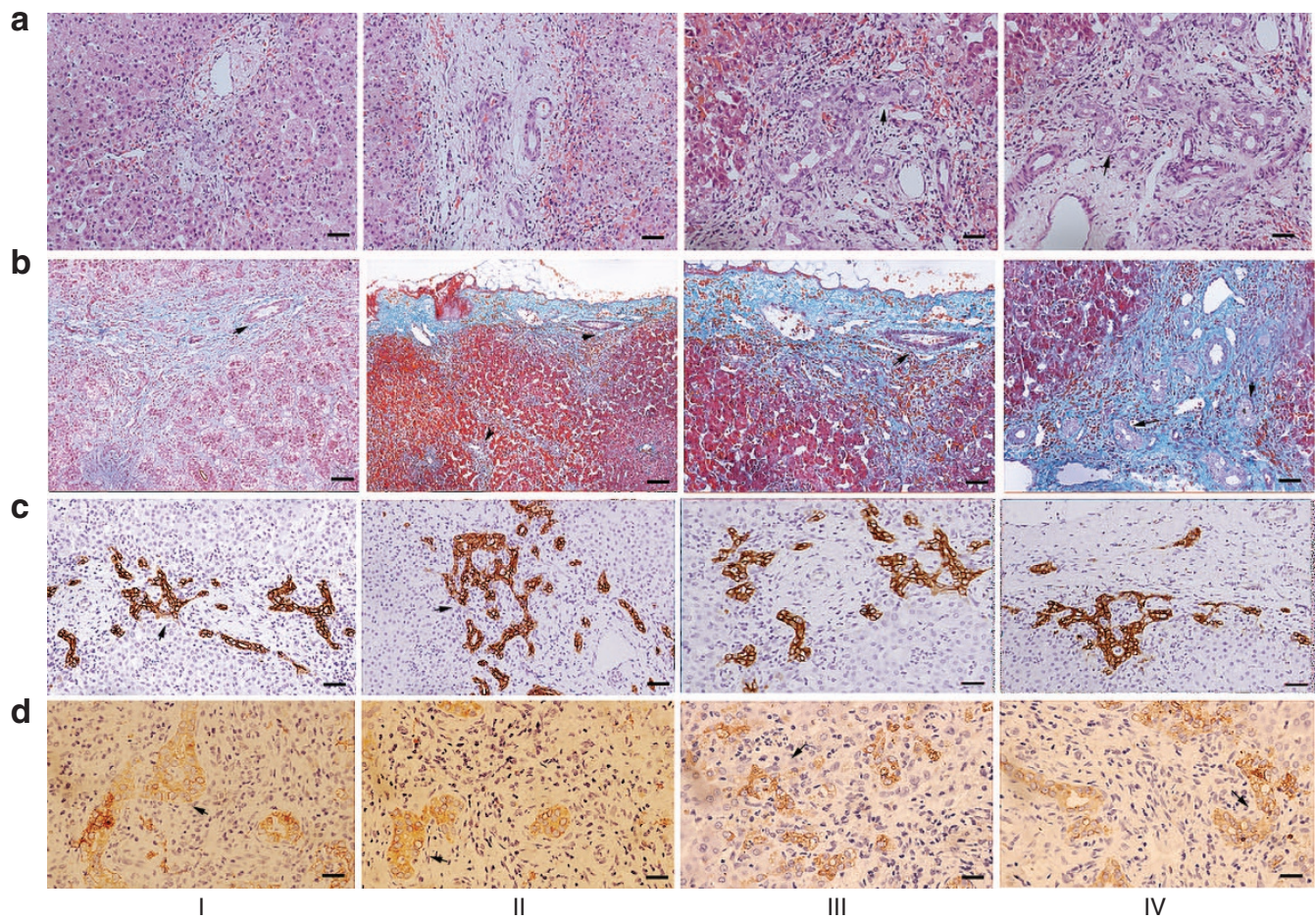

Figure 1. Representative hematoxylin \& eosin and Masson's trichrome stainings and immunohistochemical CK-7 results from biliary atresia (BA) liver sections. Cells with fibroblast-like shapes emerged within the periportal scar and within the parenchyma (a). The fibrosis grades were divided into classes from I to IV (b). Fibroblastic-like cells present around a hyperplastic cholangiocyte (c,d). Scale bars: $100 \mu \mathrm{m}(\mathbf{a}, \mathbf{b}), 50 \mu \mathrm{m}$ (c), $200 \mu \mathrm{m}$ (d).

of $\alpha$-SMA protein in the BA liver samples increased with the liver fibrosis progression (Figure 3a). In contrast, the numbers of cells that expressed $\alpha$-SMA and CK-7 were reduced with the severity of BA fibrosis (Figure 3b).

\section{miR-200b Inhibits TGF- $\beta 1$-Induced EMT in BECs}

TGF- $\beta 1$ is the most established mediator and regulator of EMT (11). Upon stimulation with TGF- $\beta 1$, BECs have been observed to undergo EMT-like transformations as evidenced by the loss of cell-cell adhesion and alterations in morphology that involve a transition from a round, compact shape to a spindle shape (Figure 4a,b). It has also been shown that BECs significantly lose the expressions of CK-7 and E-cadherin; in contrast, these cells acquire the expressions of N-cadherin, vimentin, and $\alpha$-SMA (Figure $4 c, d$ ). We have demonstrated that miR-200b can prevent TGF- $\beta 1$ induced EMT in intestinal cells (16), which suggested us that miR-200b might suppress TGF- $\beta 1$-mediated EMT in BECs. Indeed, we observed that miR-200b significantly supressed the cholangiocyte EMT by increasing the expression of E-cadherin and inhibiting the expressions of vimentin, $\mathrm{N}$-cadherin, $\alpha$-SMA, and the E-cadherin transcriptional repressor zinc finger E-box-binding homeobox (ZEB)1 (Figure 5).

\section{DISCUSSION}

It has long been recognized that the cells located within and closely around the portal areas with myofibroblastic phenotypes are the main source of fibrosis in BA $(17,18)$. However, several recent studies have suggested that some alternative mechanisms might also contribute to the expansion of myofibroblastic populations in BA $(14,15,19)$. Here, we found and confirmed evidence for EMT in BA and showed that bile duct epithelia underwent EMT and transdifferentiated into myofibroblasts; thus, these cells might be an additional and significant source of fibroblasts in BA. We showed that $\alpha$-SMA localized to both the fibroblast-like cells and the BECs and colocalized with the marker immature ductular epithelia marker CK-7 in the epithelial cells of deformed duct structures. 


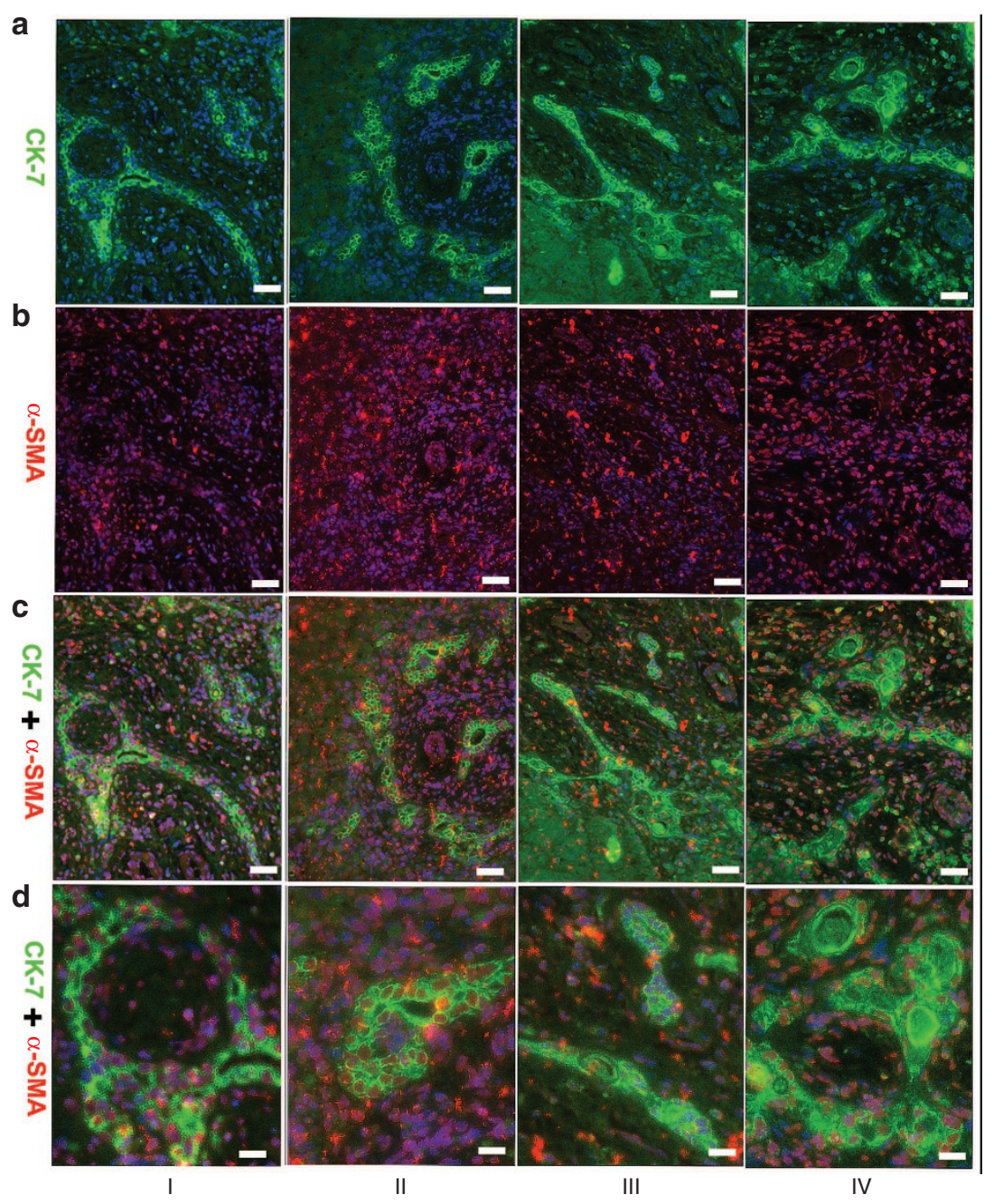

Figure 2. Evidence of biliary epithelial-mesenchymal transition (EMT) in patients with biliary atresia (BA). The mesenchymal marker $\alpha$-smooth muscle actin ( $\alpha-S M A)$ and the marker of immature biliary epithelial cells CK-7 colocalized in the ductular epithelial cells in BA. CK-7 (a), $\alpha-S M A$ (b), merge of CK-7 (green) and $\alpha$-SMA (red) (c,d). Scale bars: $100 \mu \mathrm{m}(\mathbf{a}-\mathbf{c}), 200 \mu \mathrm{m}(\mathbf{d})$.

Interestingly, the number of cells that coexpressed $\alpha$-SMA and CK-7 was reduced with the severity of BA fibrosis. The phenotypic shift that was characterized by the gain of $\alpha$-SMA expression and decreased staining for the epithelial marker CK-7 might explain the "loss" of BECs and the "gain" of portal tract fibroblasts that eventually differentiate into $\alpha$-SMA-expressing myofibroblasts.

TGF- $\beta$ is critical for the progression of fibrosis and is the major inducer of EMT (11). It has been well documented that the production of TGF- $\beta$ by BECs and surrounding cells increases in BA cases with prominent ductular reactions. Additionally, TGF- $\beta$ secretion by activated portal fibroblasts is also increased in BA and isolated cells $(18,20,21)$. Our in vitro findings suggest that cholangiocyte EMT in BA might be induced by TGF- $\beta 1$, which decreased the expressions of epithelial markers and increased the expressions of mesenchymal markers in BECs and induced morphological changes toward mesenchymal features. The members of the miR-200 family are regarded as powerful inhibitors of EMT in tumor invasion (10). Here, we found that miR-200b significantly inhibited
TGF- $\beta 1$ induction in cultured BECs via the upregulation of the expression of E-cadherin and the downregulation of the expressions of vimentin, $\alpha$-SMA, and $\mathrm{N}$-cadherin. These findings suggest that miR-200b might attenuate the progression of BA fibrosis by suppressing the cholangiocyte EMT.

In summary, our results revealed that BA fibrosis is due at least in part to biliary EMT and provide direct evidence that miR-200b inhibits EMT in BECs.

\section{METHODS}

\section{Materials}

The primary human intrahepatic BECs were obtained from PriCells (Wuhan, China). Dulbecco's modified Eagle's medium and fetal bovine serum were purchased from Gibco (Los Angeles, CA). Anti-vimentin, anti-E-cadherin, anti-N-cadherin, anti-ZEB1 and anti-glyceraldehyde 3-phosphate dehydrogenase (GAPDH) antibodies, and recombinant human TGF- $\beta 1$ were purchased from Cell Signaling Technology (Danvers, MA). Anti- $\alpha$-SMA antibody was obtained from Sigma-Aldrich (St Louis, MO). Lipofectamine RNAiMAX was purchased from Invitrogen (Carlsbad, CA). The Hasmir-200b mimics and inhibitors were synthesized by GenePharma (Shanghai, China). 
a

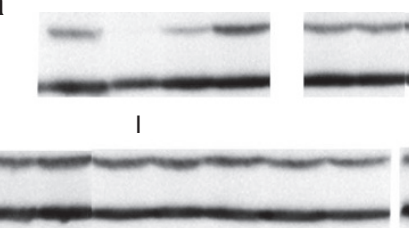

III
$\alpha-S M A$

GAPDH

II

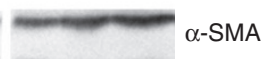

IV

b

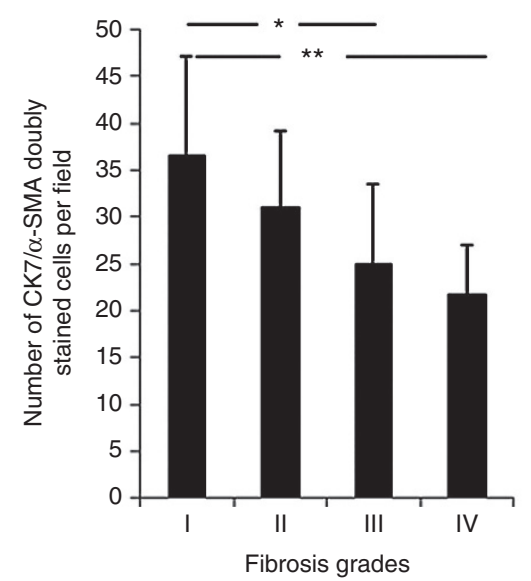

Figure 3. The expression of $\alpha$-smooth muscle actin ( $\alpha$-SMA) was related to the progression of biliary atresia (BA) fibrosis. The $\alpha$-SMA protein levels were positively associated with the severity of BA fibrosis (a), and the numbers of cells coexpressing CK-7 and $\alpha$-SMA were decreased with the severity of BA fibrosis (b). ${ }^{*} P<0.05,{ }^{* *} P<0.01$.

\section{Specimen Preparation}

A total of 18 liver biopsies were obtained from BA patients who were referred to the Xin Hua Hospital, Shanghai Jiao Tong University, Shanghai, China. This study was performed according to a protocol approved by the faculty of the Medicine Ethics Committee of Xin Hua Hospital in accordance with the ethical standards prescribed by the Helsinki Declaration of the World Medical Association. Written informed consent (XHEC-D-2012-011) was obtained from the guardians prior to specimen collection.

\section{Masson's Trichrome Staining}

Masson's trichrome staining was performed according to the manufacturer's protocol (Genmed Scientifics, Wilmington, DE). The collagen fiber was stained blue, the nuclei were stained black, and the background was stained red.

\section{Immunohistochemistry and Immunofluorescence}

The immunohistochemistry assays were performed with chromogen $3,3^{\prime}$-diaminobenzidine, which is a horseradish peroxidase substrate. Briefly, the liver sections were incubated with xylol and descending concentrations of ethanol. Endogenous peroxidases were removed with $0.3 \% \mathrm{H}_{2} \mathrm{O}_{2}$. The CK-7 antibody was then applied at an optimal concentration overnight in a wet chamber after blocking with $5 \%$ bovine serum albumin for $1 \mathrm{~h}$ at room temperature. The slides were rinsed in phosphate-buffered saline and incubated with the secondary antibody. Antibody binding was visualized with a liquid 3'-diaminobenzidine substrate Chromogen System (Dako, Glostrup, Denmark). The slides were rinsed in phosphate-buffered saline and counterstained with hematoxylin. For immunofluorescence analysis, the cells were initially fixed with $4 \%$ paraformaldehyde. The sides were then incubated with anti-CK-7 or anti- $\alpha$-SMA antibodies at room temperature for $2 \mathrm{~h}$. After washing three times with phosphatebuffered saline, the secondary antibodies conjugated to fluorescein isothiocyanate or fluorescein amidite were applied to these cells for



Figure 4. TGF- $\beta 1$-induced epithelial-mesenchymal transition (EMT) in human biliary epithelial cells (BECs) in vitro. Morphological observations revealed that the BECs lost cell-cell junctions and became scattered in respond to treatment with $10 \mathrm{ng} / \mathrm{ml}$ of TGF- $\beta 1$ (a,b). Immunofluorescence assays indicated that TGF- $\beta 1$ reduced the expression of CK-7 but induced $\alpha$-smooth muscle actin expression in BECs (c,d). Scale bar: $100 \mu \mathrm{m}(\mathbf{a}-\mathbf{d})$. 
a



b

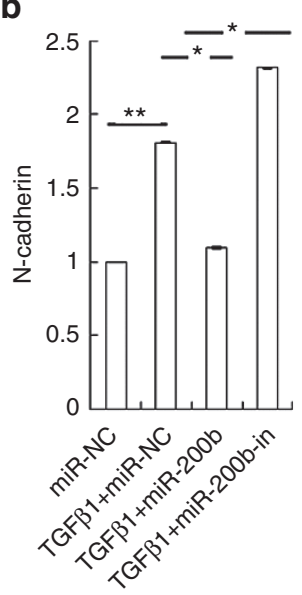

C

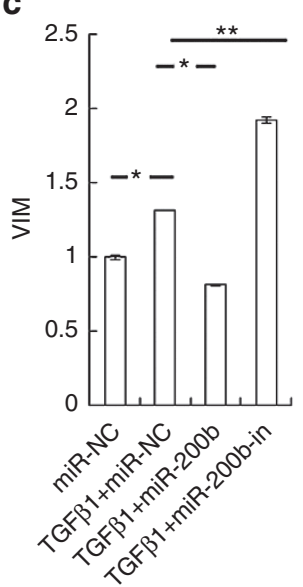

d



e

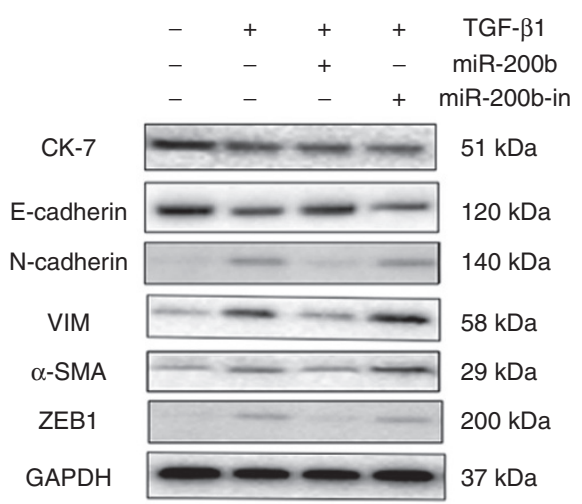

Figure 5. miR-200b inhibited TGF- $\beta 1$-induced biliary epithelial-mesenchymal transition (EMT). miR-200b significantly inhibited TGF- $\beta 1$-mediated EMT in biliary epithelial cells by increasing the expression of E-cadherin and repressing vimentin, $\alpha$-smooth muscle actin ( $\alpha$-SMA), and N-cadherin at both the mRNA and protein levels as indicated by quantitative-polymerase chain reaction and western blots, respectively. Changes in E-cadherin (a), $\mathrm{N}$-cadherin (b), vimentin (c), and $\alpha$-SMA (d) mRNAs. Western blots $(\mathbf{e}) .{ }^{*} P<0.05,{ }^{* *} P<0.01$.

$1 \mathrm{~h}$ at room temperature. The nuclei were counterstained with $4^{\prime}$, 6-diamidino-2-phenylindole.

\section{Cell Culture and Transfection}

The human intrahepatic BECs were seeded the day before transfection and grown to $50 \%$ confluence. The cells were transfected with miR-200b mimics or 200b inhibitor using lipofectamine RNAiMAX. EMT was induced with conditioned medium that was supplemented with $0.5 \%$ fetal bovine serum containing TGF- $\beta 1(10 \mathrm{ng} / \mathrm{ml})$ as described previously (5).

\section{Real-Time Polymerase Chain Reaction}

The total RNA was extracted with Trizol reagent (Invitrogen) according to the manufacturer's protocol. A SYBR-Green Universal Master Mix kit and a High Capacity cDNA Reverse Transcription kit (Applied Biosystems, Foster City, CA) were employed to detect the mRNA levels. All reactions were repeated three times. GAPDH was used to normalize the target genes. The utilized polymerase chain reaction primers were as follows: CDH1, forward, 5'-GGAGGAGAGCGGTGGTCAAA-3',

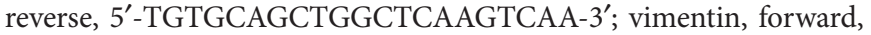
5'-CCTCCTACCGCAGGATGTT-3', reverse, 5' -CTGCCCAGGCTGT AGGTG-3'; $\alpha$-SMA, forward 5'- CCGACCGAATGCAGAAGGA-3', reverse, 5' $5^{\prime}$-ACAGAGTATTTGCGCTCCGAA-3'; N-cadherin, forward 5'-GGTGGAGGAGAAGAAGACCAG-3', reverse, 5'-GGCATCAGG CTCCACAGT- ${ }^{\prime}$; and GAPDH, forward, $5^{\prime}$-GAAGGTGAAGGTCG GAGTC-3', reverse, 5'-GAAGATGGTGATGGGATTTC-3'.

\section{Western Blot}

Western blot analyses were performed according to standard procedures using enhanced chemiluminescence detection. Briefly, the proteins were separated on $4-12 \%$ sodium dodecyl sulfatepolyacrylamide gels and then transferred onto nitrocellulose membranes using a dry blotting system (iBlot Dry Blotting System, Invitrogen). The membranes were incubated with the primary antibodies (CK-7, 1:500; E-cadherin, 1:1,000; N-cadherin, 1:1,000; ZEB1, $1: 1,000$; vimentin, $1: 1,000$; $\alpha$-SMA, $1: 2,000$; and GAPDH, $1: 1,000$ ) and secondary antibodies. After final washes with $1 \times$ phosphatebuffered saline with $0.1 \%$ Tween-20, the signals were detected with enhanced chemiluminescence chemiluminescence reagents (Pierce, Rockford, IL).

\section{Statistical Analyses}

All data are reported as the mean $\pm \mathrm{SD}$. When comparisons were made between two groups, statistical significance was determined by Student's $t$-test using the SPSS19 software program.

\section{STATEMENT OF FINANCIAL SUPPORT}

This work was supported by Shanghai Key Laboratory of Pediatric Gastroenterology and Nutrition (11DZ2260500); Shanghai Health Bureau Scientific Research (20134123); Shanghai Committee of Science and Technology (13ZR1460000); and Doctoral Innovation Fund of School Medicine, Shanghai Jiao Tong University (BXJ201328).

Disclosure: The authors certify that there are no potential perceived conflicts of interest or financial disclosures related to this work.

\section{REFERENCES}

1. Narayanaswamy B, Gonde C, Tredger JM, Hussain M, Vergani D, Davenport M. Serial circulating markers of inflammation in biliary atresia-evolution of the post-operative inflammatory process. Hepatology 2007;46:180-7.

2. Choi SS, Diehl AM. Epithelial-to-mesenchymal transitions in the liver. Hepatology 2009;50:2007-13. 
3. Sicklick JK, Choi SS, Bustamante M, et al. Evidence for epithelial3mesenchymal transitions in adult liver cells. Am J Physiol Gastrointest Liver Physiol 2006;291:G575-83.

4. Iwano M, Plieth D, Danoff TM, Xue C, Okada H, Neilson EG. Evidence that fibroblasts derive from epithelium during tissue fibrosis. J Clin Invest 2002;110:341-50.

5. Zeisberg M, Yang C, Martino M, et al. Fibroblasts derive from hepatocytes in liver fibrosis via epithelial to mesenchymal transition. J Biol Chem 2007;282:23337-47.

6. Omenetti A, Porrello A, Jung Y, et al. Hedgehog signaling regulates epithelial-mesenchymal transition during biliary fibrosis in rodents and humans. J Clin Invest 2008;118:3331-42.

7. Gregory PA, Bracken CP, Smith E, et al. An autocrine TGF-beta/ZEB/ miR-200 signaling network regulates establishment and maintenance of epithelial-mesenchymal transition. Mol Biol Cell 2011;22:1686-98.

8. Gregory PA, Bert AG, Paterson EL, et al. The miR-200 family and miR-205 regulate epithelial to mesenchymal transition by targeting ZEB1 and SIP1. Nat Cell Biol 2008;10:593-601.

9. KorpalM,LeeES, Hu G, Kang Y.ThemiR-200 familyinhibits epithelial-mesenchymal transition and cancer cell migration by direct targeting of E-cadherin transcriptional repressors ZEB1 and ZEB2. J Biol Chem 2008;283:14910-4.

10. Park SM, Gaur AB, Lengyel E, Peter ME. The miR-200 family determines the epithelial phenotype of cancer cells by targeting the E-cadherin repressors ZEB1 and ZEB2. Genes Dev 2008;22:894-907.

11. Zavadil J, Böttinger EP. TGF-beta and epithelial-to-mesenchymal transitions. Oncogene 2005;24:5764-74.

12. Chen Y, Xiao Y, Ge W, et al. miR-200b inhibits TGF-ß1-induced epithelialmesenchymal transition and promotes growth of intestinal epithelial cells. Cell Death Dis 2013;4:e541.
13. Deng YH, Pu CL, Li YC, et al. Analysis of biliary epithelial-mesenchymal transition in portal tract fibrogenesis in biliary atresia. Dig Dis Sci 2011;56:731-40.

14. Harada K, Sato Y, Ikeda H, et al. Epithelial-mesenchymal transition induced by biliary innate immunity contributes to the sclerosing cholangiopathy of biliary atresia. J Pathol 2009;217:654-64.

15. Díaz R, Kim JW, Hui JJ, et al. Evidence for the epithelial to mesenchymal transition in biliary atresia fibrosis. Hum Pathol 2008;39:102-15.

16. Flier SN, Tanjore H, Kokkotou EG, Sugimoto H, Zeisberg M, Kalluri R. Identification of epithelial to mesenchymal transition as a novel source of fibroblasts in intestinal fibrosis. J Biol Chem 2010;285: 20202-12.

17. Shirahase I, Ooshima A, Tanaka K, Yamabe H, Inomata $\mathrm{Y}$, Ozawa K. Immunohistochemical demonstration of collagen types III and IV and myofibroblasts in the liver of patients with biliary atresia. J Pediatr Surg 1994;29:639-44.

18. Ramm GA, Nair VG, Bridle KR, Shepherd RW, Crawford DH. Contribution of hepatic parenchymal and nonparenchymal cells to hepatic fibrogenesis in biliary atresia. Am J Pathol 1998;153:527-35.

19. Omenetti A, Bass LM, Anders RA, et al. Hedgehog activity, epithelialmesenchymal transitions, and biliary dysmorphogenesis in biliary atresia. Hepatology 2011;53:1246-58.

20. Milani S, Herbst H, Schuppan D, Stein H, Surrenti C. Transforming growth factors beta 1 and beta 2 are differentially expressed in fibrotic liver disease. Am J Pathol 1991;139:1221-9.

21. Lamireau T, Le Bail B, Boussarie L, et al. Expression of collagens type I and IV, osteonectin and transforming growth factor beta-1 (TGFbeta1) in biliary atresia and paucity of intrahepatic bile ducts during infancy. J Hepatol 1999;31:248-55. 